\title{
Coherent "Metallic" Resistance and Medium Localization in a Disordered One-Dimensional Insulator
}

\author{
Martin Moško, ${ }^{1,2}$ Pavel Vagner, ${ }^{2,1}$ Michal Bajdich, ${ }^{1}$ and Thomas Schäpers ${ }^{2}$ \\ ${ }^{1}$ Institute of Electrical Engineering, Slovak Academy of Sciences, Dúbravská cesta 9, 84104 Bratislava, Slovakia \\ ${ }^{2}$ Institut für Schichten und Grenzflächen, Forschungszentrum Jülich GmbH, 52425 Jülich, Germany
}

(Received 12 November 2002; published 26 September 2003)

It is believed that a disordered one-dimensional (1D) wire with coherent electronic conduction is an insulator with the mean resistance $\langle\rho\rangle \simeq e^{2 L / \xi}$ and resistance dispersion $\Delta_{\rho} \simeq e^{L / \xi}$, where $L$ is the wire length and $\xi$ is the electron localization length. Here we show that this $1 \mathrm{D}$ insulator undergoes at full coherence the crossover to a 1D "metal," caused by thermal smearing and resonant tunneling. As a result, $\Delta_{\rho}$ is smaller than unity and tends to be $L / \xi$ independent, while $\langle\rho\rangle$ grows with $L / \xi$ first nearly linearly and then polynomially, manifesting the so-called medium localization.

DOI: $10.1103 /$ PhysRevLett.91.136803

PACS numbers: 73.23.-b, 71.23.An, 73.61.Ey

Any coherent electron wave in a one-dimensional (1D) wire with uncorrelated disorder is exponentially localized [1]. The resistance $\rho$ of such a disordered 1D wire should therefore increase exponentially with the wire length, $L$. In an ensemble of macroscopically identical wires the resistance fluctuates from wire to wire owing to disorder randomness, so it is natural to study the mean resistance $\langle\rho\rangle$, where the angular brackets denote the average over the ensemble. Landauer found at zero temperature [2]

$$
\langle\rho\rangle=0.5[\exp (2 L / \xi)-1],
$$

where $\xi$ is the electron localization length (throughout this Letter, $\rho$ is a dimensionless resistance in units $h / 2 e^{2}$ ). He also found that $\langle 1 / \rho\rangle$ diverges and noted that $\langle\rho\rangle$ is not representative of the ensemble. Anderson et al. [3] later defined the typical resistance $\rho_{t} \equiv$ $\exp \langle f\rangle-1$, where $f=\ln (1+\rho)$. For $T=0 \mathrm{~K}$ they found $\langle f\rangle=L / \xi$, i.e.,

$$
\rho_{t}=\exp (L / \xi)-1
$$

The variable $\rho$ exhibits giant fluctuations, the dispersion $\Delta_{\rho} \equiv\left(\left\langle\rho^{2}\right\rangle-\langle\rho\rangle^{2}\right)^{1 / 2} /\langle\rho\rangle$ can be shown to grow as [4]

$$
\Delta_{\rho} \simeq \exp (L / \xi) .
$$

However, $\Delta_{f} \propto 1 / \sqrt{L}$, which means that the variable $f$ self-averages and $\rho_{t}$ is representative of the ensemble [3].

The above formulas hold also for a quasi-1D wire with many 1D channels [5,6]. Thus, for $T=0 \mathrm{~K}$ and $L>\xi$ any $1 \mathrm{D}$ wire is insulating, i.e., both the resistance and fluctuations grow exponentially with $L / \xi$.

Thouless [5] argued that this exponential rise would not be apparent at $T>0 \mathrm{~K}$ since the inelastic collisions would cause electrons to hop from one localized state to another. This would cause $\rho \propto L$ and the 1D localization would be manifested solely by a typical temperature dependence of hopping, whenever the transport time through the wire exceeds the inelastic scattering time.

At even higher temperatures, the electron coherence length $L_{\phi}$ would become smaller than $\xi$. This would give rise to the "metallic" resistance $(\rho \simeq L / \xi)$ and weak localization (see, e.g., Ref. [7]). The crossover between the metallic resistance and hopping, first predicted in Ref. [5], has been observed in the quasi-1D wires [8].

Therefore, the disordered 1D insulator described by Eqs. (1)-(3) is believed to exist at appropriately low temperatures, i.e., without hopping and at full coherence [7,9], and the crossover to the 1D "metal" seems to occur only if inelastic collisions are present.

We show that the crossover to another 1D metal occurs at full coherence due to the thermal smearing and resonant tunneling. It results in the wire resistance rising with $L / \xi$ first nearly linearly and then polynomially owing to the medium localization. In addition, $\Delta_{\rho}$ becomes smaller than unity and tends to be $L / \xi$ independent.

The insulator-metal transition at $T=0 \mathrm{~K}$ has recently been found in a 1D solid with specially correlated disorder [10]. In our work the disorder is uncorrelated and the reported insulator-metal crossover, albeit also coherent, is driven by low nonzero temperatures.

We consider a 1D wire with disorder described by potential $V(x)=\sum_{j=1}^{N} \gamma \delta\left(x-x_{j}\right)$, where $\gamma \delta\left(x-x_{j}\right)$ is the $\delta$-shaped impurity potential of strength $\gamma, x_{j}$ is the $j$ th impurity position randomly chosen along the wire, and $N$ is the number of impurities. If the $1 \mathrm{D}$ electrons tunnel through disorder coherently, the electron wave function $\Psi_{k}(x)$ is the solution of the tunneling problem

$$
\begin{gathered}
{\left[-\frac{\hbar^{2}}{2 m} \frac{d^{2}}{d x^{2}}+V(x)\right] \Psi_{k}(x)=\varepsilon \Psi_{k}(x),} \\
\Psi_{k}(x \rightarrow 0)=e^{i k x}+r_{k} e^{-i k x}, \quad \Psi_{k}(x \rightarrow L)=t_{k} e^{i k x},
\end{gathered}
$$

where $\varepsilon=\hbar^{2} k^{2} / 2 m$ is the electron energy, $k$ is the wave vector, $m$ is the effective mass, $r_{k}$ is the electron reflection amplitude, and $t_{k}$ is the electron transmission amplitude. In the boundary conditions (5) the electron impinging disorder from the left is considered. The beginning and end of the wire are set at $x=0$ and $x=L$, respectively. 
The transfer matrix (TM) of disorder $V(x)$ reads [11]

$$
\mathbb{T}=\left(\begin{array}{cc}
1 / t_{k}^{*} & -r_{k}^{*} / t_{k}^{*} \\
-r_{k} / t_{k} & 1 / t_{k}
\end{array}\right)=\mathbb{T}\left(x_{N}\right) \ldots \mathbb{T}\left(x_{2}\right) \mathbb{T}\left(x_{1}\right),
$$

where

$$
\mathbb{T}\left(x_{j}\right)=\left(\begin{array}{cc}
1-i \Omega / k & -i \frac{\Omega}{k} e^{-2 i k x_{j}} \\
i \frac{\Omega}{k} e^{2 i k x_{j}} & 1+i \Omega / k
\end{array}\right)
$$

is the TM of the $\delta$ barrier at position $x_{j}$ and $\Omega=m \gamma / \hbar^{2}$. We evaluate the right-hand side of Eq. (6) numerically.

We then obtain the transmission probability $\mathcal{T}=\left|t_{k}\right|^{2}$ and evaluate numerically the two-terminal conductance

$$
G=\int_{0}^{\infty} d \varepsilon\left[-\frac{d f(\varepsilon)}{d \varepsilon}\right] \mathcal{T}(\varepsilon),
$$

where $f(\varepsilon)$ is the Fermi distribution. To calculate the wire resistance, instead of $\rho=1 / G$ we use the formula [12]

$$
\rho=1 / G-1 / f(0),
$$

where $1 / f(0)$ is the contact resistance $(\simeq 1$ at low $T)$. At zero temperature Eq. (9) gives $\rho=\mathcal{R}\left(\varepsilon_{F}\right) / \mathcal{T}\left(\varepsilon_{F}\right)$, where $\mathcal{R}=1-\mathcal{T}$ and $\varepsilon_{F}$ is the Fermi energy. Equations (1)(3) were derived $[2-4,6]$ by averaging the formula $\rho=$ $\mathcal{R}\left(\varepsilon_{F}\right) / \mathcal{T}\left(\varepsilon_{F}\right)$. We average Eq. (9) since $T>0 \mathrm{~K}$.

We parametrize disorder by parameters $\mathcal{R}_{I}\left(k_{F}\right)$ and $N_{I}$, where $\mathcal{R}_{I}(k)=\Omega^{2} /\left(k^{2}+\Omega^{2}\right)$ is the reflection probability for a single $\delta$ barrier and $N_{I}$ is the 1D impurity density. Note that the final results do not depend on the choice of $\mathcal{R}_{I}(k)$ as we assume very small $k_{B} T / \varepsilon_{F}$. For $\mathcal{R}_{I}\left(k_{F}\right) \ll 1$ we can also ignore fluctuations of $\mathcal{R}_{I}\left(k_{F}\right)$ from impurity to impurity.

In Fig. 1 we show the averaged 1D resistance versus the wire length and temperature. The results were obtained for disorder with parameters $\mathcal{R}_{I}\left(k_{F}\right)=0.01$ and $N_{I}=$ $10^{7} \mathrm{~m}^{-1}$ and for the electron gas parameters typical of the GaAs wire: $m=0.067 m_{0}$ and $\varepsilon_{F}=35 \mathrm{meV}$.

In fact, for $\mathcal{R}_{I}\left(k_{F}\right) \ll 1$ and $N_{I}^{-1} \gg 2 \pi / k_{F}$ (weak lowdensity disorder) our results depend only on the parameters $L / \xi$ and $T / T_{\xi}$, independently on the choice of $\mathcal{R}_{I}\left(k_{F}\right), N_{I}, m$, and $\varepsilon_{F}$. Here $k_{B} T_{\xi}=1 /\left[g\left(\varepsilon_{F}\right) \xi\right]$, where $g\left(\varepsilon_{F}\right)=1 /\left(\pi \hbar v_{F}\right)$ is the density of energy levels. For weak low-density disorder the localization length is just the elastic mean free path, i.e., $\xi=\left[N_{I} R_{I}\left(k_{F}\right)\right]^{-1}$ [3]. For the above parameters $\xi=10 \mu \mathrm{m}$ and $T_{\xi} \simeq 1 \mathrm{~K}$.

In the top panel of Fig. 1 we reproduce at $T / T_{\xi}=0$ the exponential growth (1) and (2). However, at $T / T_{\xi}>0$ both $\langle\rho\rangle$ and $\rho_{t}$ tend to grow with $L / \xi$ much slower than predicted by Eqs. (1) and (2). At $T / T_{\xi}>1$ they exhibit up to $L / \xi \leq 2$ the metallic dependence $\langle\rho\rangle=\rho_{t}=L / \xi$ with a nonlinear correction which is $\leq 0.4 L / \xi$. For larger $L / \xi$ this nonlinear growth is still far much slower than $\exp (L / \xi)$ and in general not $\exp ($ const $L$ ) (see Fig. 3).

The bottom panel of Fig. 1 shows the dispersions $\Delta_{\rho}$ and $\Delta_{g}$, where $g=1 / \rho$. At $T=0 \mathrm{~K}, \Delta_{\rho}$ follows the
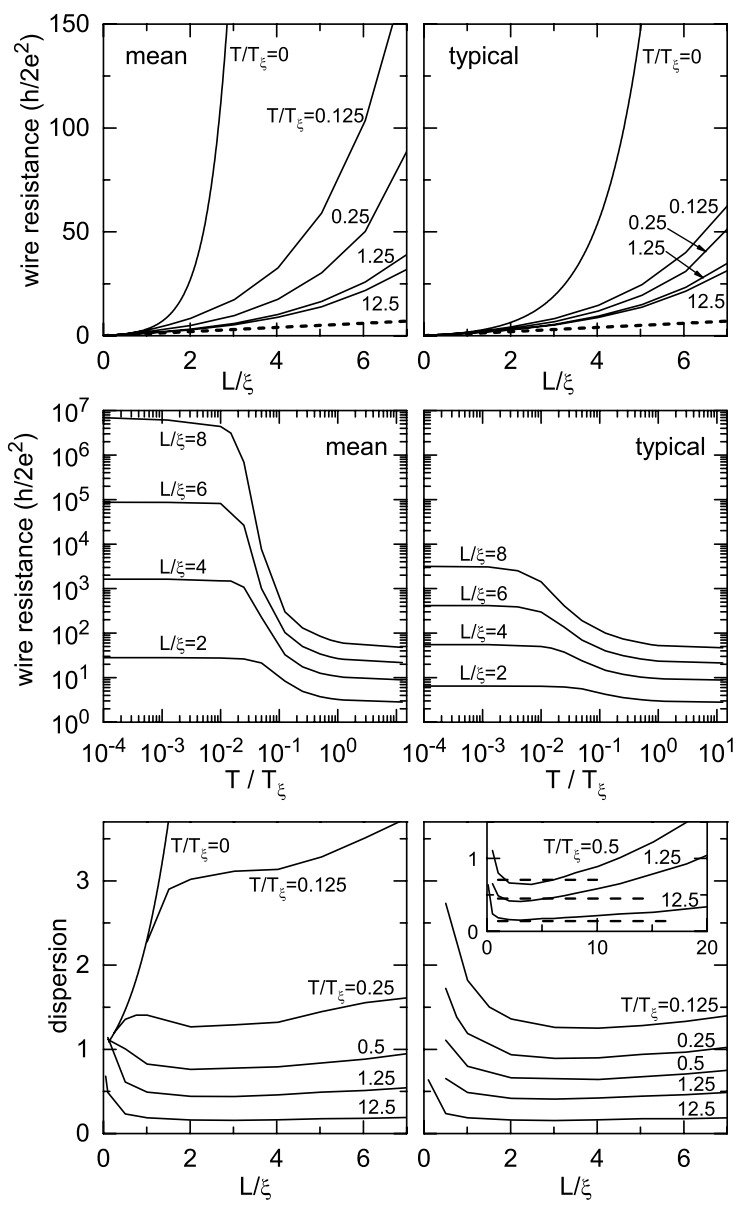

FIG. 1. Top panel: Mean resistance $\langle\rho\rangle$ and typical resistance $\rho_{t}$ vs normalized length $L / \xi$ for various normalized temperatures $T / T_{\xi}$. The dashed line is the metallic dependence $\rho=$ $L / \xi$. Middle panel: $\langle\rho\rangle$ and $\rho_{t}$ vs $T / T_{\xi}$ for various $L / \xi$. Bottom panel: Dispersion of the resistance (left) and conductance (right) vs $L / \xi$ for various $T / T_{\xi}$. The inset shows the comparison with the estimate $0.5 \sqrt{T_{\xi} / T}$ (dashed line) derived in the text.

exponential rise (3) and $\Delta_{g}$ diverges. As $T$ increases, $\Delta_{\rho}$ and $\Delta_{g}$ decrease below unity and variation with $L$ tends to disappear, unlike other transport regimes in which $\Delta_{\rho}$ and $\Delta_{g}$ always vary with $L$ in some typical way [7].

To provide insight we now approximate Eq. (8) by

$$
G \simeq \frac{1}{4 k_{B} T} \int_{\varepsilon_{F}-2 k_{B} T}^{\varepsilon_{F}+2 k_{B} T} d \varepsilon \mathcal{T}(\varepsilon) .
$$

A typical $\mathcal{T}(\varepsilon)$ curve (Fig. 2) consists of narrow peaks separated on average by energy $k_{B} T_{L}=1 /\left[g\left(\varepsilon_{F}\right) L\right]$. Some of these peaks dominate due to the resonant tunneling, the rest are the much lower peaks with a negligible area compared to the area below the resonant peaks. The mean distance between the resonant peaks is $k_{B} T_{\xi}$ and the mean number of such peaks in the energy window $4 k_{B} T$ is $4 k_{B} T / k_{B} T_{\xi}$ [13]. Thus, for $T \gtrsim T_{\xi}$ we can estimate the mean and variance of the integral (10) as

136803-2 

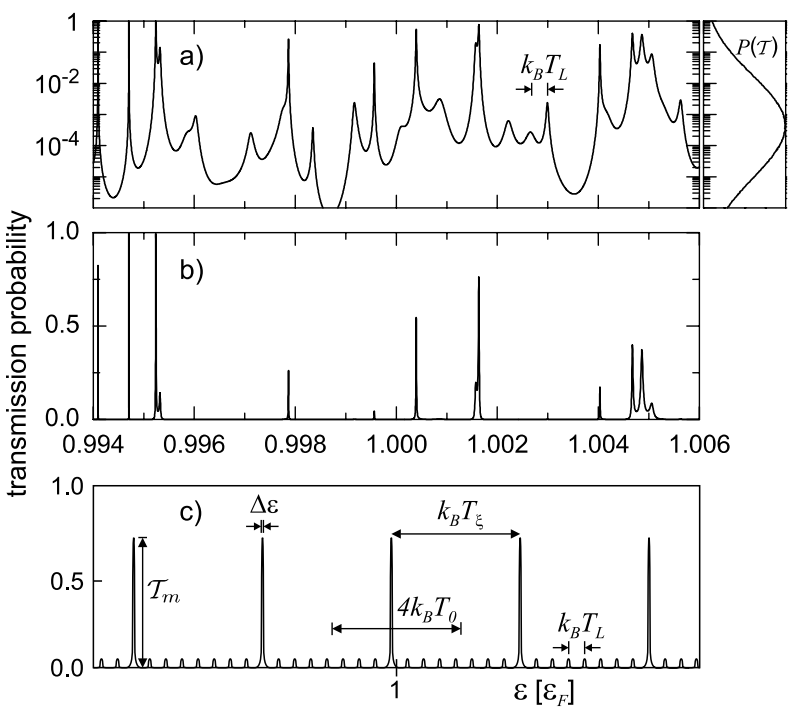

FIG. 2. (a) Transmission probability $\mathcal{T}(\varepsilon)$ versus energy $\varepsilon$ for one random configuration of disorder and distribution $P(\mathcal{T})$ averaged over many configurations. Energy axis is in units $\varepsilon_{F}$, where $\varepsilon_{F}=35 \mathrm{meV}$. The length parameters are $L / \xi=8$ and $L=80 \mu \mathrm{m}$, other parameters are the the same as quoted for Fig. 1. (b) The $\mathcal{T}(\varepsilon)$ dependence from (a) in linear scale. (c) Schematic generalization of (a),(b) (see the text).

$$
\langle G\rangle \simeq \frac{\mathcal{T}_{m} \Delta \varepsilon}{4 k_{B} T} \frac{4 k_{\mathrm{B}} T}{k_{B} T_{\xi}}, \quad \sqrt{\operatorname{var}(G)} \simeq \frac{\mathcal{T}_{m} \Delta \varepsilon}{4 k_{B} T} \sqrt{\frac{4 k_{B} T}{k_{\mathrm{B}} T_{\xi}}},
$$

where $\mathcal{T}_{m}$ and $\Delta \varepsilon$ are the average height and width of the resonant peak. Equations (11) give the $L$-independent dispersion $\Delta_{G} \simeq 0.5 \sqrt{T_{\xi} / T}$, in Fig. 1 compared with the simulated $\Delta_{g}$. A small difference between $g$ and $G$ is not essential here, the estimate and simulation differ because the $\operatorname{var}(G)$ in Eq. (11) ignores deviations from $\mathcal{T}_{m} \Delta \varepsilon$.

At $T \gtrsim T_{\xi}$ thermal averaging causes $\langle 1 / G\rangle \simeq 1 /\langle G\rangle$. Then $\langle\rho\rangle=\langle 1 / G\rangle-1 \simeq 1 /\langle G\rangle$ for not too small $L / \xi$. To illustrate how the coherent wire becomes more metallic than insulating, we set in Eq. (11) the "ballistic" $\operatorname{maxima} \mathcal{T}_{m}=1$ and $\Delta \varepsilon=k_{B} T_{L}$. We obtain $\langle G\rangle \simeq$ $T_{L} / T_{\xi}=\xi / L$ and $\langle\rho\rangle \simeq L / \xi$. This metal exists if the window $4 k_{B} T$ involves at least one resonant peak. This happens for $4 T / T_{\xi} \geq 1$, i.e., the crossover temperature is $T_{0}=0.25 T_{\xi}$.

For $T>T_{0}$ we see in the middle panel of Fig. 1 a tendency to the $T$ independence, in accord with the $T$-independent $\langle G\rangle$ of Eq. (11). Generally, $\langle\rho\rangle=1 /$ $\langle G\rangle-1$ for $T \gg T_{0}$, but $\langle G\rangle$ is $T$ independent for any $T$ :

$$
\langle G\rangle=\int_{0}^{\infty} d \varepsilon\left[-\frac{d f(\varepsilon)}{d \varepsilon}\right]\langle\mathcal{T}(\varepsilon)\rangle \simeq \int_{0}^{1} d \mathcal{T} \mathcal{T} P(\mathcal{T})
$$

where $P(\mathcal{T})$ is the distribution of transmission $\mathcal{T}$ (Fig. 2).

Figure 3 shows $\langle\rho\rangle$ and $\rho_{t}$ from Fig. 1 in semilog scale. For $T>T_{\xi}$ both $\langle\rho\rangle$ and $\rho_{t}$ rise with $L / \xi$ nonlinearly, but far much more slowly than $\exp (L / \xi)$. Is this rise of the form $\exp (\operatorname{const} L)$ ? It is not at least for $L / \xi \lesssim 12$. The
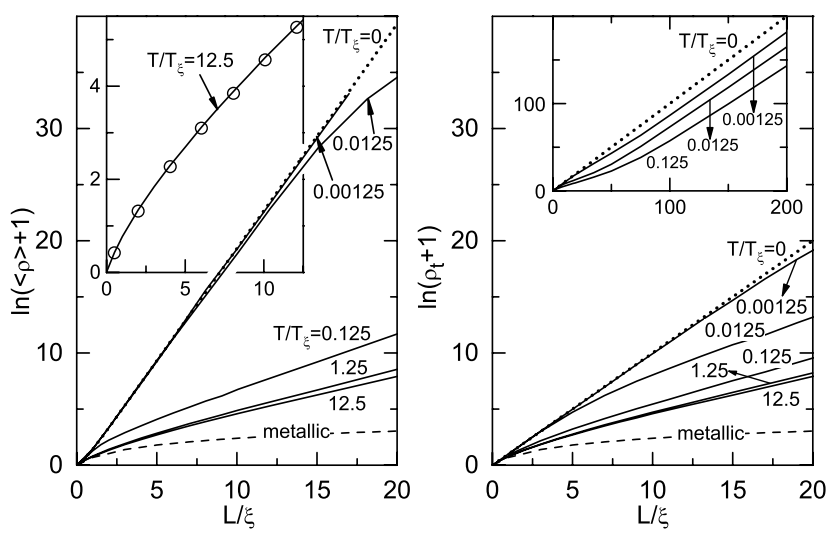

FIG. 3. Mean resistance and typical resistance versus $L / \xi$ for various $T / T_{\xi}$. The dashed line is the metallic resistance $\rho=$ $L / \xi$. Inset to the left panel: The $T=12.5 T_{\xi}$ curve is shown in more detail to stress the sublinear concave shape. The open circles show the limit $\langle\rho\rangle=\langle G\rangle^{-1}-1$, where $\langle G\rangle$ is given by Eq. (12) with $P(\mathcal{T})$ generated numerically. Inset to the right panel: The typical resistance simulated up to $L / \xi=200$, but only for $T / T_{\xi} \leq 0.125$ owing to huge computational time.

inset to the left panel clearly shows that the rise is sublinear in semilog scale. Since $\langle\rho\rangle \rightarrow \rho_{t}$, we continue in terms of $\rho_{t}$, which is defined by $\ln \left(1+\rho_{t}\right) \equiv\langle\ln (1+\rho)\rangle$. Obviously, equation $\langle\ln (1+\rho)\rangle=$ const $\times L$ cannot fit the sublinear curve in the inset. The resistance thus rises with $L$ polynomially rather than exponentially. This is neither the strong localization [the SL is manifested by Eqs. (1)-(3)] nor the weak localization (the WL occurs at $L / \xi \ll 1)$, but somewhere between. We therefore speak about the medium localization, which means a nonexponential decay of resonant transmission with $L / \xi$.

What happens for extremely large $L / \xi$ ? The inset to the right panel shows the typical resistance for $T \ll T_{\xi}$. Here the rise $\propto \exp (L / \xi)$ reappears at $L / \xi \gtrsim 50$. For $T>T_{\xi}$ neither $\rho_{t}$ nor $\langle\rho\rangle$ is numerically feasible, but for $L / \xi \geq 40$ we find $\rho_{t} \simeq\langle\rho\rangle \propto(L / \xi)^{3 / 2} \exp (L / 4 \xi)$ by using another approach not reported here. In the "infinite" wire also the resonant transmission is finally damped exponentially.

Note, however, that the limit of extremely long coherent $1 \mathrm{D}$ segments is experimentally irrelevant. In general, the resistance $\geq 10^{9} \Omega$ is hardly measurable owing to intrinsic physical limitations (e.g., the rf noise [8]). On the insulating side of the crossover, one can thus measure $\rho_{t} \simeq e^{L / \xi}$ in principle for $L / \xi \lesssim 12$. On the metallic side, both $\langle\rho\rangle$ and $\rho_{t}$ reach $10^{9} \Omega$ for $L / \xi \simeq 30$ (not shown in Fig. 3), so they could be measurable for $L / \xi \lesssim 30$.

In Ref. [14] the resistance of a single 1D channel was measured for various $L>\xi$ in a series of GaAs quantum wires prepared by a method allowing to reproduce macroscopic parameters from wire to wire. In Fig. 4 the experiment [14] is compared with our simulation. In inset (a) we compare the two-terminal resistances. We see that the measured data reasonably reflect the superlinear rise of the theoretical curve. Inset (b) proves that the superlinear 


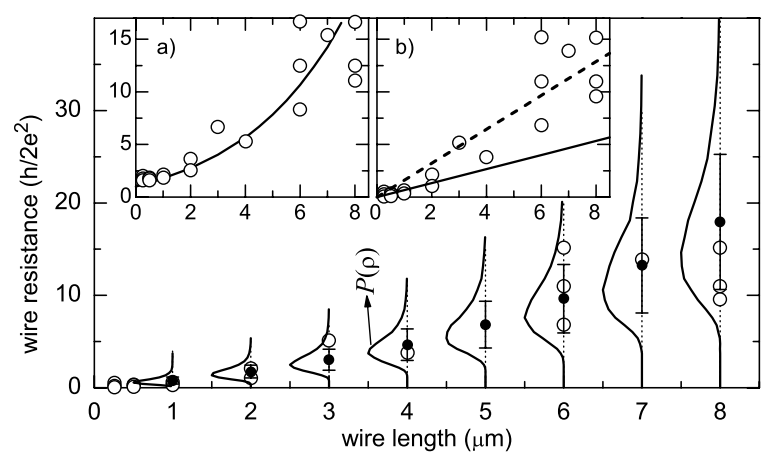

FIG. 4. (a) Open circles are the two-terminal resistance data of Ref. [14], measured at $T=4.2 \mathrm{~K}$ in a series of GaAs quantum wires with a single occupied channel. In these wires $\xi \simeq 1.5 \mu \mathrm{m}$ and $\varepsilon_{F} \simeq 3.5 \mathrm{meV}$, as the quantized ballistic conductance is observed for $L \lesssim 1.5 \mu \mathrm{m}$ and the lowest quantized step is centered roughly at the mentioned $\varepsilon_{F}$ value [14]. For these parameters $T_{\xi} \simeq 2 \mathrm{~K}$. The full line shows the simulated mean two-terminal resistance, $\langle 1 / G\rangle$. In our simulation the value $\xi \simeq 1.5 \mu \mathrm{m}$ can be realized through many different combinations of parameters $N_{I}$ and $R_{I}\left(k_{F}\right)$, but the results remain the same provided the disorder is weak. So we do not need to consider sample-dependent details. (b) Open circles are the experimental data from inset (a) reduced by the contact resistance (the two-terminal resistance at $L \rightarrow 0$ ). To prove the superlinear rise with $L$ clearly, the experimental data are compared with the linear fit $\rho=L / \xi$. The best fit is obtained for $\xi=0.6 \mu \mathrm{m}$ (dashed line). However, it overestimates all experimental data for $L \leq 2 \mu \mathrm{m}$ and the value $\xi=0.6 \mu \mathrm{m}$ is in contradiction with the ballistic conductance observed for $L \lesssim 1.5 \mu \mathrm{m}$ [14]. For $\xi=1.5 \mu \mathrm{m}$ (full line) the fit is indeed good for the ballistic wire lengths, but for $L>2 \mu \mathrm{m}$ the experimental data grow superlinearly. Main panel: The calculated mean resistance $\langle\rho\rangle$ (full circles), standard deviation from $\langle\rho\rangle$ (vertical bars), and resistance distribution $P(\rho)$ are shown. Open circles are the same as those in inset (b).

rise of the measured data is systematically above $\rho=$ $L / \xi$ and cannot be ascribed to the data dispersion. The main panel shows that the simulated mean resistance, standard deviation and resistance distribution fit [12] the experiment. For a perfect comparison experiments with very large ensembles of wires are needed.

If decoherence would be present in the experiment [14], it would cause the dependence $\rho=L / \xi$, which is not observed. This means that in the measured 1D wires $L_{\phi} \gtrsim 8 \mu \mathrm{m}$, which is an order of magnitude more than in a related 2D electron gas at the same temperature [7]. Why is $L_{\phi}$ so large? At low temperatures decoherence is due to the electron-electron interaction. In the (ballistic) 2D electron gas the interaction of two electrons fulfills the conservation laws $\varepsilon\left(k_{1}\right)+\varepsilon\left(k_{2}\right)=\varepsilon\left(k_{1}^{\prime}\right)+\varepsilon\left(k_{2}^{\prime}\right)$ and $k_{1}+k_{2}=k_{1}^{\prime}+k_{2}^{\prime}$, which allow the energy exchange and decoherence. In the 1D system such conservation laws prohibit any energy exchange, which might be the reason for large $L_{\phi}$. One might think [9] that $L_{\phi} \lesssim \xi$ since the diffusion is controlled by exponential localization. In our case $L_{\phi}$ can exceed $\xi$ many times as the resistance of segment $L_{\phi}$ grows with $L_{\phi} / \xi$ weakly superlinearly, not exponentially. A full calculation of $L_{\phi}$ in the 1D wire is beyond the Fermi-liquid theory and has not yet appeared.

In summary, due to the thermal smearing and resonant tunneling, the disordered 1D insulator shows at full coherence the crossover to the 1D metal. The resistance of the 1D metal grows with $L / \xi$ first nearly linearly and then polynomially due to the medium localization. This is in contrast to the expectation that the resistance of the coherent 1D system grows with $L / \xi$ exponentially if $L / \xi>1$. The $1 \mathrm{D}$ metal shows the resistance dispersion which is almost $L / \xi$ independent and smaller than unity, again in contrast with the expected (exponential) growth. Such 1D metal should be observable in any coherent 1D system of length $L / \xi \lesssim 30$, longer coherent segments are experimentally irrelevant. The crossover temperature is $T_{0}=0.25 T_{\xi}$, in the GaAs wires $T_{0} \simeq 0.1-1 \mathrm{~K}$.

M. M. was supported by the APVT Agency Grant No. APVT-20-021602. P.V. was supported by the EC Grant No. HPMFCT-2000-00702 and VEGA Grant No. 2/3118/23.

[1] N. F. Mott and W. D. Twose, Adv. Phys. 10, 107 (1961).

[2] R. Landauer, Philos. Mag. 21, 863 (1970).

[3] P.W. Anderson et al., Phys. Rev. B 22, 3519 (1980).

[4] P. A. Mello, Phys. Rev. B 35, 1082 (1987).

[5] D. J. Thouless, Phys. Rev. Lett. 39, 1167 (1977).

[6] P.W. Anderson, Phys. Rev. B 23, 4828 (1981).

[7] S. Datta, Electronic Transport in Mesoscopic Systems (Cambridge University Press, Cambridge, U.K., 1995).

[8] M. E. Gershenson et al., Phys. Rev. Lett. 79, 725 (1997).

[9] Y. Imry, Introduction to Mesoscopic Physics (Oxford University Press, Oxford, U.K., 2002).

[10] F. M. Izrailev et al., Phys. Rev. Lett. 82, 4062 (1999).

[11] J.H. Davies, The Physics of Low-Dimensional Semiconductors: An introduction (Cambridge University Press, Cambridge, U.K., 1998).

[12] The two-terminal resistance $1 / G$ includes the contact resistance, while Eq. (9) is the resistance of disorder, measurable by four probes. Equation (9) ignores the effect of measurement probes [M. Büttiker, IBM J. Res. Dev. 32, 72 (1988)], but one can see that for disordered wires the problem is not serious. First, if presented in terms of $1 / G$, the mean resistance and resistance distribution in Fig. 4 remain the same except for a constant shift by the value of the contact resistance (the $1 / G$ at $L \rightarrow 0$ ). Second, the resistance of disorder can be well estimated from the measured $1 / G$ by subtracting the contact resistance.

[13] M. Ya. Azbel and D. P. DiVincenzo, Phys. Rev. B 30, 6877 (1984), studied the same regime at temperatures too low $\left(T \ll T_{\xi}\right)$ to see the effects discussed by us.

[14] D. Kaufman et al., Phys. Rev. B 59, R10 433 (1999). 